\title{
Effect of deletion of gene cluster involved in synthesis of Enterobacterial common antigen on virulence and immunogenicity of live attenuated Salmonella vaccine when delivering heterologous Streptococcus pneumoniae antigen PspA
}

\author{
Qing Liu ${ }^{1,2+}$, Xuegang Shen ${ }^{1+}$, Xiaoping Bian ${ }^{1}$ and Qingke Kong ${ }^{1 *}$ (D)
}

\begin{abstract}
Background: Enterobacterial common antigen (ECA) is a family-specific surface antigen shared by all members of the Enterobacteriaceae family. Previous studies showed that the loss of ECA results in Salmonella attenuation, indicating its usefulness as a vaccine candidate for Salmonella infection, but no studies have shown whether the mutation resulting from the deletion of the ECA operon in conjunction with other mutations could be used as an antigen vehicle for heterologous protein antigen delivery.
\end{abstract}

Results: In this study, we introduced a nonpolar, defined ECA operon deletion into wild-type S. Typhimurium x3761 and an attenuated vaccine strain X9241, obtaining two isogenic ECA operon mutants, namely, X12357 and X12358, respectively. A number of in vitro and in vivo properties of the mutants were analyzed. We found that the loss of ECA did not affect the growth, lipopolysaccharide (LPS) production and motility of S. Typhimurium wild type strain X3761 and its attenuated vaccine strain X9241 but significantly affected the virulence when administered orally to $\mathrm{BALB} / \mathrm{c}$ mice. Furthermore, the effects of the ECA mutation on the immunogenicity of a recombinant $S$. Typhimurium vaccine strain $\times 9241$ when delivering the pneumococcal antigen PspA were determined. The result showed that the total anti-PspA IgG level of X12358 (pYA4088) was slightly lower than that of X9241 (pYA4088), but the protection rate was not compromised.

Conclusions: ECA affects virulence and benefits the Th2 immunity of Salmonella Typhimurium, therefore, it is feasible to use a reversible ECA mutant mode to design future Salmonella vaccine strains for heterologous protective antigens.

Keywords: ECA, Salmonella Typhimurium, Virulence, Vaccine vector, Th2 immunity

\footnotetext{
* Correspondence: kongqiki@163.com

${ }^{\dagger}$ Qing Liu and Xuegang Shen contributed equally to this work.

'College of Animal Science and Technology, Southwest University, Chongqing, China

Full list of author information is available at the end of the article
}

(c) The Author(s). 2020 Open Access This article is licensed under a Creative Commons Attribution 4.0 International License, which permits use, sharing, adaptation, distribution and reproduction in any medium or format, as long as you give appropriate credit to the original author(s) and the source, provide a link to the Creative Commons licence, and indicate if changes were made. The images or other third party material in this article are included in the article's Creative Commons licence, unless indicated otherwise in a credit line to the material. If material is not included in the article's Creative Commons licence and your intended use is not permitted by statutory regulation or exceeds the permitted use, you will need to obtain permission directly from the copyright holder. To view a copy of this licence, visit http://creativecommons.org/licenses/by/4.0/ The Creative Commons Public Domain Dedication waiver (http://creativecommons.org/publicdomain/zero/1.0/) applies to the data made available in this article, unless otherwise stated in a credit line to the data. 


\section{Background}

Enterobacterial common antigen (ECA) is a kind of unique glycolipid on the cell surface of all Enterobacteriaceae family members, such as Klebsiella, Proteus, Shigella, Yersinia, and Salmonella [1-4]. It consists of linear repetitive units of a trisaccharide composed of 4-acetamide-4,6dideoxy-D-galactose (Fuc4NAc), N-acetyl-D-mannosaminuronic acid (ManNAcA), and N-acetyl-D-glucosamine (GlcNAc). It is considered the second dominant immunogen, ranked next to the lipopolysaccharide (LPS) Oantigen $[3,5,6]$. Three ECA variants, $\mathrm{ECA}_{\mathrm{PG}}, \mathrm{ECA}_{\mathrm{LPS}}$ and $\mathrm{ECA}_{\mathrm{CYC}}$, have been described since it was first found in $E$. coli $[3]$. ECA $\mathrm{EG}_{\mathrm{PG}}$ is the major form that is linked to diacylglycerol and the only type of ECA present in Salmonella [7]; $\mathrm{ECA}_{\mathrm{LPS}}$ is anchored to the lipid A core region of LPS [8]; and $\mathrm{ECA}_{\mathrm{CYC}}$, which contains four or six trisaccharide repeat units, is a water-soluble cyclic form, not expressed on the surface of bacteria but in the periplasmic space [2, 9]. ECA plays important roles in virulence in the Enterobacteriaceae family because of its impacts on bile resistance, motility and other characteristics [10-13]. Similar with $\mathrm{O}$-antigen, ECAs are also polysaccharide antigens anchored on the surface of bacteria cells and can trigger the production of anti-ECA antibodies in mice. Nevertheless, these anti-ECA antibodies hardly account for the passive immune protection against these Enterobacteriaceae strains, which distinguish from other virulence factors and surface polysaccharides. Furthermore, these anti-ECA antibodies can escape from the host immune system and last over a long period of time in the bodies of hosts, such as patients with shigellosis, peritonitis [14], chronic urinary tract infections (UTIs) [15] or chronic pyelonephritis [14-16]. The avoidance of triggering anti-ECA antibody production in the host by the Salmonella vaccine may contribute to increased stimulation of the immune response against heterologous antigens.

The genetic determinants of ECA $\mathrm{PG}_{\mathrm{PG}}$ are located in the wec (formerly $r f e$ ) gene cluster from wecA to wecG (Fig. 1a), which is responsible for the synthesis of ECA polysaccharides, the addition of ECA polysaccharide chains to the lipid carrier and the transportation of ECA to the bacterial cell surface $[11,17,18]$. The inactivation of specific genes in this gene cluster results in the loss of ECA polysaccharides in Enterobacteriaceae family members and attenuates these pathogens. For example, the wecA mutation in Haemophilus ducreyi 35000HP impairs pustule formation in humans, and the wecE mutation in uropathogenic E. coli (UPEC), which results in dysfunction in the synthesis of ECAs, attenuates the effects of murine urinary tract infection $[19,20]$. In Salmonella Typhimurium, $\Delta w e c A$ mutants are also more sensitive to bile and less lethal to mice. Due to the undesirable host immune response stimulated by ECAs, recent studies have revised the ECA of the Salmonella vaccine carrier to deliver $\mathrm{O}$-antigen polysaccharide [21, 22]. We demonstrated that downregulation of $\mathrm{O}$-antigen expression could reduce the immune response against specific O-antigen of attenuated Salmonella carriers while triggering a strong immune response against heterologous antigens [23-25], and downregulation of both O-antigen and ECA production in Salmonella enhances the immunogenicity and cross-protective efficacy against heterologous Salmonella challenge [26]. Therefore, we speculate that the removal of ECA from live attenuated Salmonella Typhimurium vaccine strains would expose more heterologous antigens and outer membrane proteins to the host, leading to a greater immune response to the heterologous antigens and better protective efficacy.

The ideal live Salmonella vaccine carriers show attenuated virulence but an enhanced immune response against the heterologous antigens delivered by vaccine carriers. In our previous studies, a regulated delayed expressed system (RDES) in a Recombinant live recombinant attenuated Salmonella vaccine (RASV) strain was designed to deliver heterologous antigens regulated by arabinose. In this RDES, there are two components: RASV X9241-carrying mutated genes of $\triangle p a b A \triangle p a b B \triangle a s d A \Delta$ relA198::araC $\mathrm{P}_{\mathrm{BAD}}$ lacI TT deletion/insertion (TT stands for transcriptional terminator) and a plasmid pYA4088 that consist of a recombinant Streptococcus pneumoniae antigen pspA gene fused to DNA encoding the $\beta$-lactamase signal sequence for directing recombinant PspA to the periplasmic region [27]. As an attenuated live vaccine strain, X9241(pYA4088) invades and colonizes host tissues by oral immunization. The presence of arabinose positively regulates the synthesis of LacI, which can prevent PspA expression by binding to the $\mathrm{P}_{\text {trc }}$ promoter on pYA4088. In contrast, no LacI is expressed without arabinose in the growth environment of strain $X 9241$ (pYA4088), resulting in production of the heterologous PspA that are carried on pYA4088. This regulated delayed expressed system has been widely used to evaluate the effects of other mutations on immunogenicity against heterologous antigens [28-30]. In this work, we constructed an ECA-deficient Salmonella Typhimurium mutant by deleting the entire ECA operon from wecA to wecG based on the wild type strain X3761 [31] and the attenuated live vaccine strain $X 9241$ [32], and evaluated the effect of removing the whole ECA operon on the virulence and immunogenicity of wild type Salmonella Typhimurium and its attenuated mutants. We also tested the protective efficacy of the generated mutants in BALB/C mice infected with Streptococcus pneumoniae.

\section{Results}

Construction of the ECA-deficient Salmonella mutant strain

In the $S$. Typhimurium wild type strain UK-1 $\chi^{3761}$ and its attenuated vaccine strain $X 9241$, the ECA operon 


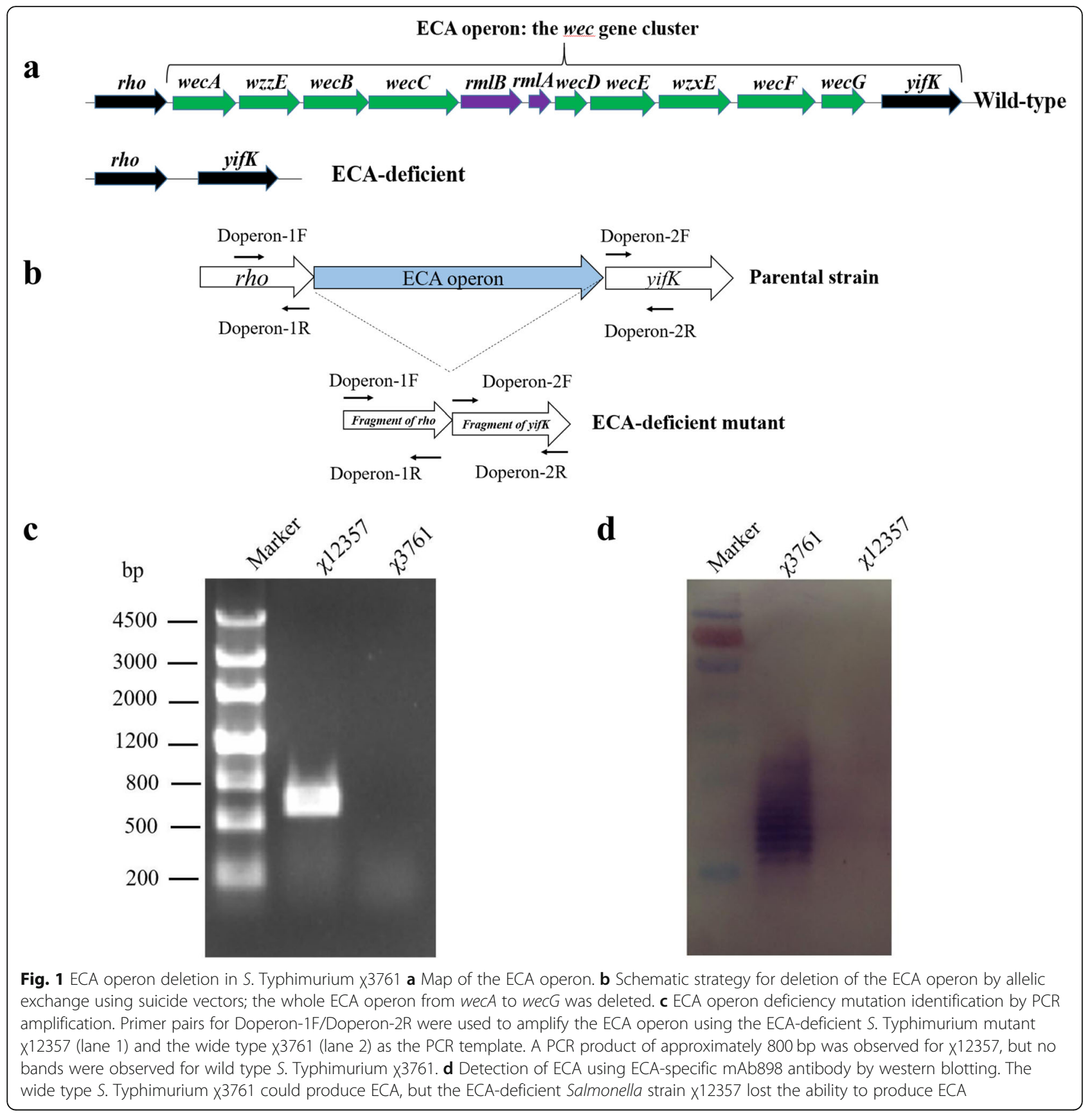

consisted of a series of wec genes, ranging from $w e c A$ to $w e c G$, as stated in Fig. 1a. We deleted the whole ECA operon of the wild type strain UK-1 X3761 and the attenuated vaccine strain $X 9241$ and generated two ECA defective mutants, $\chi 12357$ and $\chi 12358$, by suicide plasmid-mediated homologous recombination (Fig. 1b). A pair of primers, Doperon-1F/Doperon-2R, were used to amplify the ECA operon from $\times 3761$ and $\times 12357$, and a PCR product of approximately $800 \mathrm{bp}$ could be observed for $\mathrm{X} 12357$, but no bands were observed for
X3761 in agarose gel, which indicated that mutants with deletion of the ECA operon (from wecA to wecG) were created in X12357 (Fig. 1c). Furthermore, immunoblotting using the mouse anti-ECA monoclonal antibody mAb898 demonstrated deletion of the EAC operon, showing the ECA-negative phenotype [33] (Fig. 1d). Following the same method, we also deleted the ECA operon in the attenuated vaccine strain X9241 and created an ECA defective vaccine strain X12358 (data not shown). 
Phenotypic characterization of the ECA deficient mutants The growth rate of the mutants was evaluated in LB broth and showed that the growth curves of the ECA-deficient mutants $\chi 12357$ and $\chi 12358$ were similar to those of their parental strains $\chi^{3761}$ and $\chi 9241$. Both $\chi^{3761}$ and $\chi_{12357}$ reached the end of the log phase with an $\mathrm{OD}_{600}$ of 1.5 for approximately $5 \mathrm{~h}$, while the vaccine strain $\chi 9241$ and its derivative strain $\chi 12358$ had slightly lower rates of growth due to the deletion of a few virulent genes, such as $p a b A, p a b B$, $\operatorname{araBAD}$ and relA (Fig. 2a). The LPS profile of the ECA-deficient mutant $\mathrm{X} 12357$ was indistinguishable

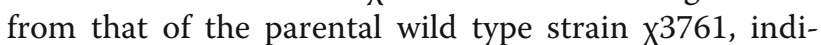
cating that ECA deficiency has no effect on the synthesis of LPS in $S$. Typhimurium (Fig. 2b). To determine the effect of ECA deficiency on bile resistance, drops of serial dilutions of $\chi^{3761}$ and $X 12357$ strain suspensions were incubated on LB plates with or without $1 \%$ deoxycholate (the main active ingredient of bile, DOC). The growth state of X3761 and X12357 appeared similar on regular LB plates (plates 2 and 3). A few colonies were observed on the $10^{7}$ dilution drops of both strain suspensions, suggesting no differences in the growth rate and susceptibility between the two strains on LB plates. Nevertheless, their growth states were totally different on the LB plates containing 1\% DOC (plate 1). For the wild type strain $\times 3761$, a few colonies grew on the $10^{7}$ dilution, and the same growth tendency as that of the regular LB plate was observed, suggested that $1 \%$ DOC had no effect on the growth of the wild type strain; however, the $10^{5}$ dilution of the $X^{12357}$

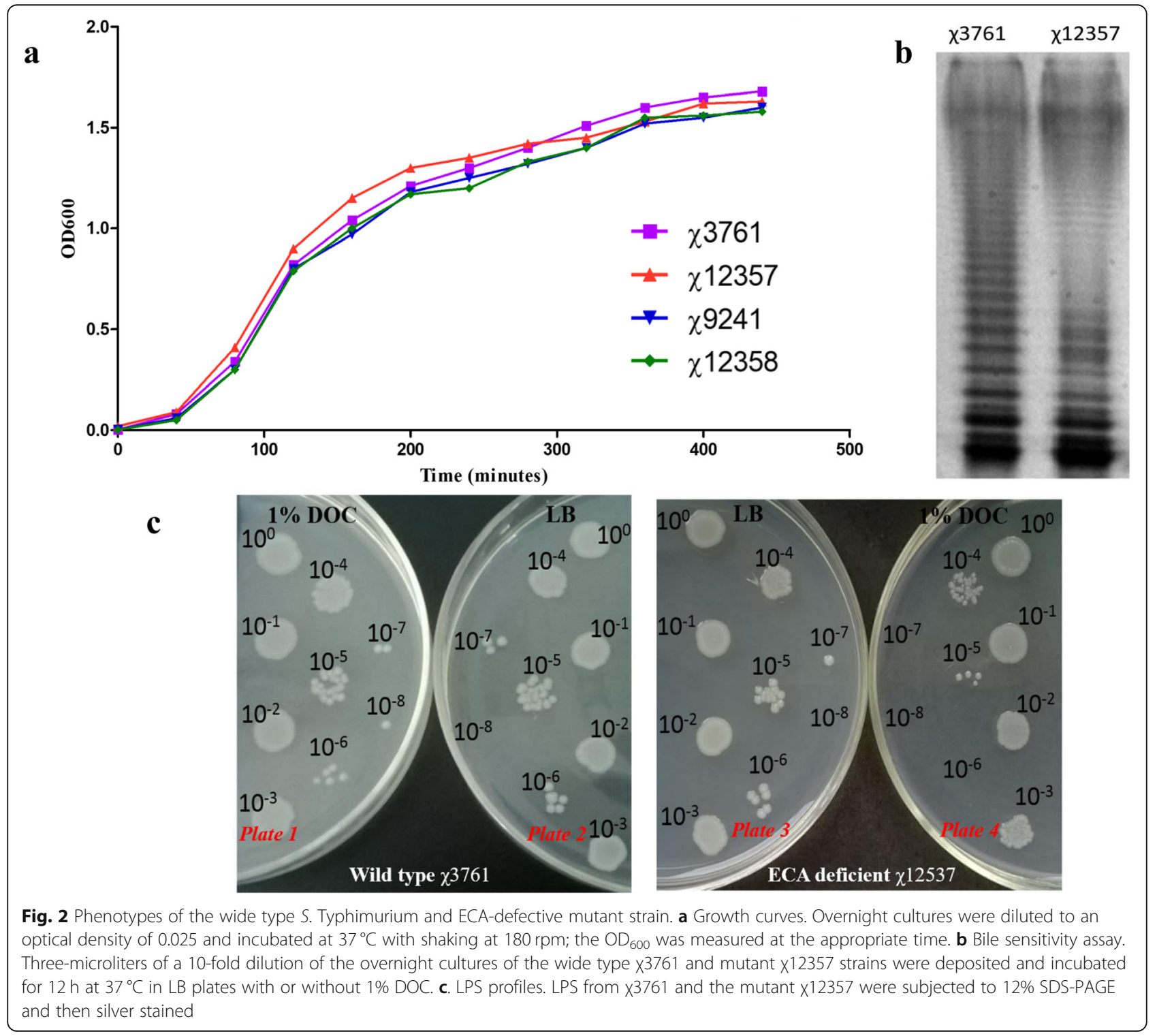


suspension grew a few colonies, suggesting that the wec operon mutants were significantly suppressed by the presence of $1 \%$ DOC on plate 4 (Fig. 2c). Moreover, under the high concentration DOC bile condition, $87.6 \%$ of the wec operon $\chi^{12357}$ mutants were also killed, but more than $90 \%$ of the wild type strains remained viable (Table 3 ). To assess the relationship between ECA and motility, the ECA-deficient mutant $\chi^{12357}$ and wild type $\chi^{3761}$ strain were dropped onto $0.3 \%$ agar plates. Both the ECAdeficient mutants and wild type strains were able to swim on soft agar, with no defects in motility (Table 1).

\section{Virulence and colonization of the mutant strain in mice}

The virulence of the ECA-deficient mutant was assessed by determining the oral $\mathrm{LD}_{50}$ of the strains in $\mathrm{BALB} / \mathrm{c}$ mice. The results showed that $\chi 3761$ was highly virulent with an $\mathrm{LD}_{50}$ of $5 \times 10^{4} \mathrm{CFU}$, whereas the ECA-deficient mutant X12357 was highly attenuated and no deaths were observed following infection of the mice at the highest tested dose $\left(1.0 \times 10^{9} \mathrm{CFU}\right)$, which suggested that the wec operon mutation made the wild type $S$. Typhimurium avirulent (Table 1). Further, the capability of the ECA-deficient mutant X12357 to efficiently disseminate and colonize mouse tissues was assessed by determining the bacterial loading in the organs of the mice, including the liver, spleen and Peyer's patch, post-infection (Fig. 3a, b, c). Consistent with the previous result, all of the mice inoculated with $x 3761$ succumbed to infection within 9 days post-infection, and all of the mice inoculated with $X 12357$ survived until 21 days post-infection and most of them appeared health and active. The colonization data showed that the bacterial counts in the organs from the mice inoculated with the wildtype strain rapidly increased, and bacterial loading in the organs of the wild-type-inoculated mice was significantly higher than that of the mice inoculated with the ECA-deficient mutant within 9 days post-infection. Further, the ECA-deficient mutant also colonized well in the mice organs and maintained a moderate level of bacterial loading, with persistent infection throughout the experiment. These data demonstrate that the ECA-deficient mutant was able to efficiently colonize the mice and establish a persistent infection, consistent with the results of a previous study [18].

Table 1 Motility, DOC sensitivity and virulence of the wild-type S. Typhimurium $\times 3761$ and its ECA-deficient mutant

\begin{tabular}{llll}
\hline Strain & Motility $(\mathrm{cm})$ on soft agar & Death rate (\%) (DOC) & $L_{50}$ \\
\hline X3761 & $6.2 \pm 0.1$ & 9.5 & $2 \times 10^{4}$ \\
X12357 & $6.4 \pm 0.1$ & 87.6 & $>10^{9}$ \\
\hline
\end{tabular}

Expression of the pneumococcal antigen PspA in the Salmonella strains

Attenuated Salmonella X9241 carrying $\triangle p a b A \triangle p a b B$ mutations is often used in our lab to evaluate the effects of other mutations on immunogenicity [24]. To assess the effects of ECA deletion on immunogenicity in attenuated Salmonella, we introduced the wec operon muta-

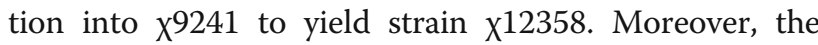
asd recombinant plasmid pYA4088, which carries a recombinant $p s p A$ gene fused to the DNA encoding the $\beta$ lactamase signal sequence to direct PspA to the periplasmic region, was introduced into both $X 9241$ and $X 12358$. Both strains ( $\chi 9241$ and $\chi 12358$ ) were grown in LB medium with or without $0.1 \%$ arabinose, and western blot analyses were used to determinate the synthesis levels of PspA in the above bacterial cells. No PspA was detected in $X 9241$ (pYA3493, carrying the empty vector, which served as a negative control) regardless of whether it was grown in the presence of arabinose. Compared with the negative control strain $X 9241$ (pYA3493), both strains ( $\chi 9241$ and $\chi^{12358)}$ carrying pYA4088 produced PspA when arabinose was absent, and instead expressed LacI in the presence of arabinose (Supplementary Fig. 1). This result suggests two points: the regulated delayed expressed system was successfully used to construct the attenuated vaccine vector strains $X 9241$ (pYA4088) and $\chi^{12358}$ (pYA4088); and the wec operon mutant $\chi^{12358}$ has a similar ability to express heterologous antibodies, such as PspA, as its parent strain $\chi^{9241 .}$

\section{Immunogenicity of the wec operon mutants after oral administration}

To assess the immunogenicity of the wec operon mutants expressing heterologous antigens as vaccine vectors, three groups of mice were immunized and boosted with $1 \times 10^{9}$ of X12358 (pYA4088), X9241 (pYA4088) or X9241 (pYA3493). Serum IgG, IgG1 and IgG2a antibody responses to PspA in sera collected at week 4 and week 6 were measured by ELISA. Compared with the negative control strain $X 9241$ (pYA3493), both $\chi^{12358}$ (pYA4088) and X9241 (pYA4088) significantly stimulated mice to produce high levels of IgG, IgG1 and IgG2a anti-PspA antibodies, suggesting that the deletion of the wec operon had no effect on the immunogenicity of the vaccine strains. Noticeably, X12358 (pYA4088) induced levels of anti-PspA IgG1 isotype antibodies that were significantly higher than that of $X^{9} 241$ (pYA4088) $(P<0.001)$, but the levels of total IgG and IgG2a isotype antibodies in the mice induced by $\mathrm{X}^{12358}$ (pYA4088) were significantly lower than that induced by X9241 (pYA4088) (Fig. 4a, b, c).

\section{Evaluation of immunogenicity against conserved OMPs from different enteric bacteria}

To evaluate the cross-reactivity of the serum antibodies raised from mice orally immunized with the mutant 


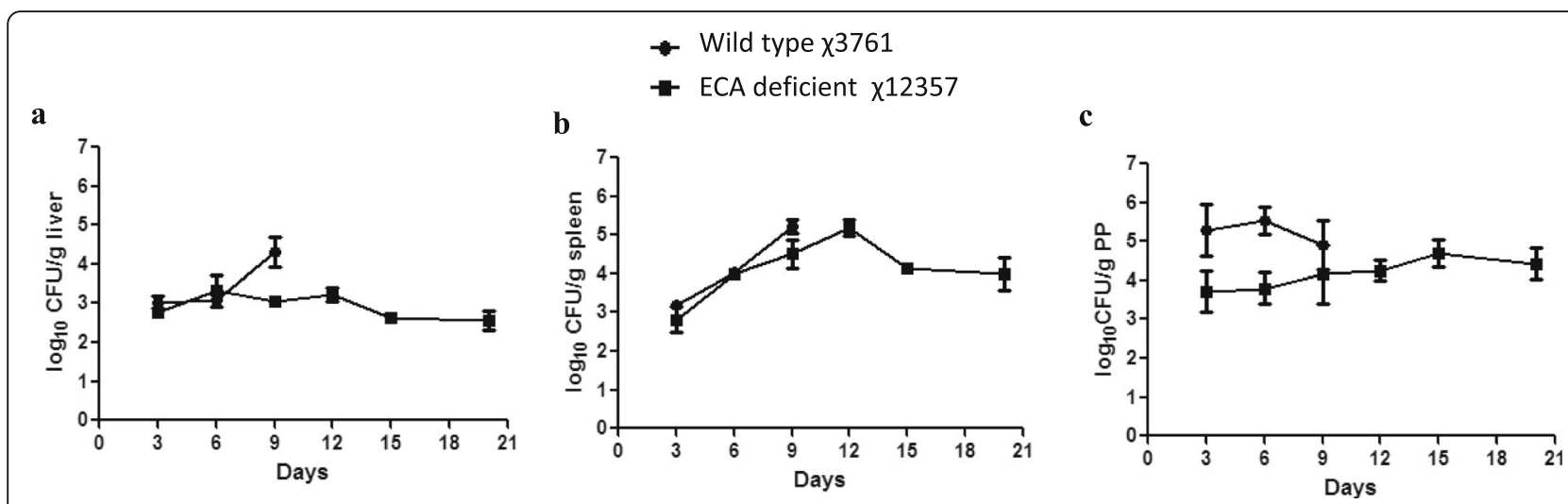

Fig. 3 Bacterial colonization in mouse organs. Groups of mice (3 mice/group) were orally inoculated with approximately $1 \times 10^{9} \mathrm{CFU}$ of the indicated strains. The bacterial loadings (log CFU/g) in the tissue were determined on days 3, 6, 9, and 12 post-inoculation in the liver (a), spleen (b), and PP (c). The horizontal lines represent the means, and the error bars represent the standard errors of the means. All the mice in wild-type X3761 group died at 9 days after oral inoculation

X12358 (pYA4088) against outer membrane proteins (OMPs) purified from several different homologous and heterologous wild-type enteric bacterial strains, including $S$. Typhimurium (group B), S. Choleraesuis (group C1), E. coli O78 and S. Enteritidis (group D1), we measured the IgG antibody responses to OMPs from the above pathogens in serum collected at week 6 after the mice were immunized with $\chi^{12358}$ (pYA4088) and $X 9241$ (pYA4088). The IgG level of cross-reaction against OMPs from S. Typhimurium (group B), E. coli O78 and $S$. Enteritidis (group D1) stimulated by $X 9241$ (pYA4088) and $\chi 12358$ (pYA4088) were not different, but the level of IgG against OMPs from $S$. Choleraesuis stimulated by the wec mutant $X 12358$ were significantly higher than that induced by $X 9241$ (pYA4088) (Fig. 5). This result was consistent with a previous study, in which a lack of or the regulated synthesis of dominant surface antigens, such as the O-antigen of LPS, increased the serum antibody cross-reactive to conserved surface OMPs of other enteric bacteria $[24,36]$.

\section{Comparison of the protective efficacy of live attenuated vaccine strains and their wec operon mutants}

To test whether the wec operon mutation affects the protective efficacy of RASVs against pneumococcal infection, BALB/c mice orally immunized and boosted with $X^{9241}$ (pYA4088) and $\chi^{12358 ~(p Y A 4088) ~ w e r e ~}$ challenged i.p. with $2.0 \times 10^{4} \mathrm{CFU}$ (50 times of $\mathrm{LD}_{50}$ ) of wild type $S$. pneumoniae WU2. No mice in the negative

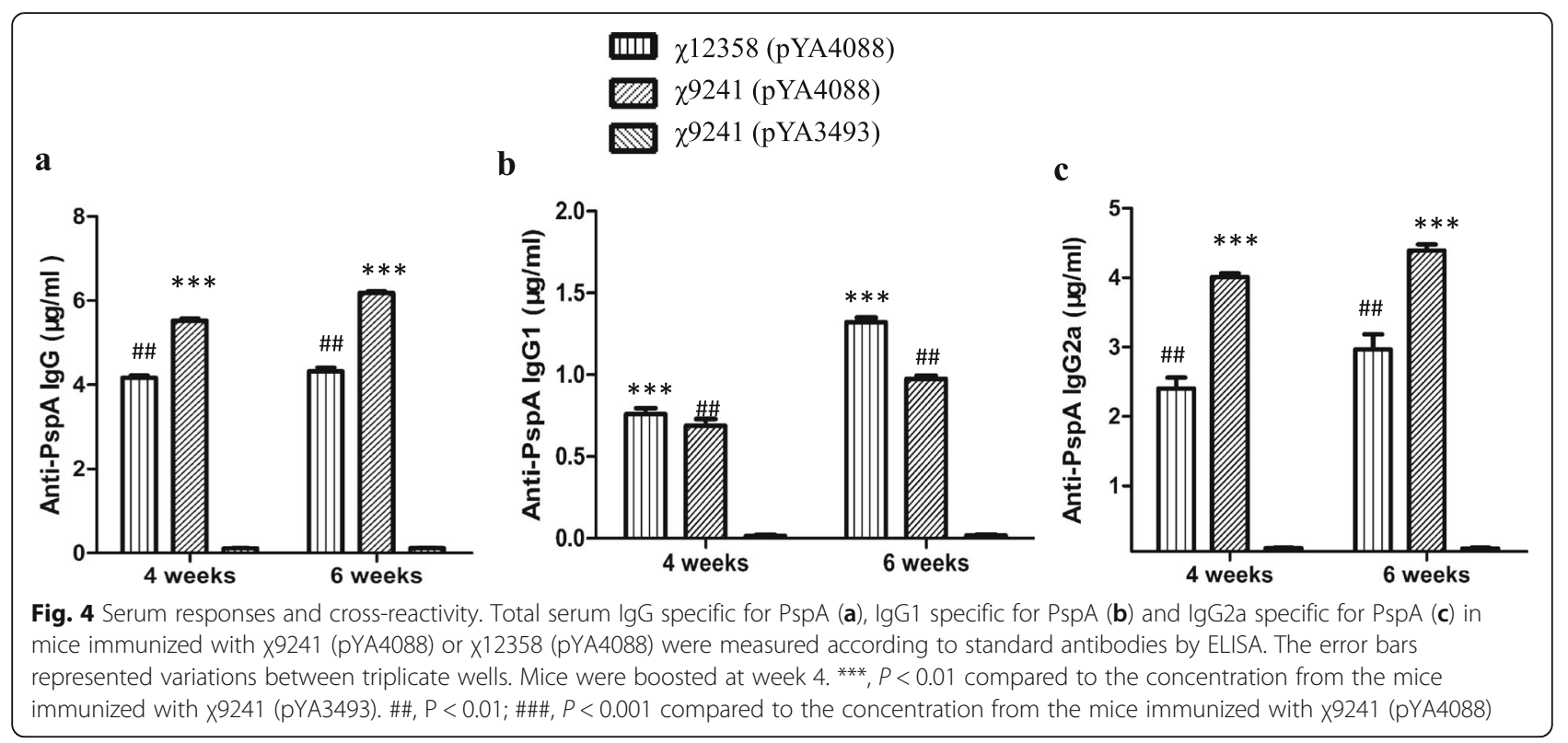




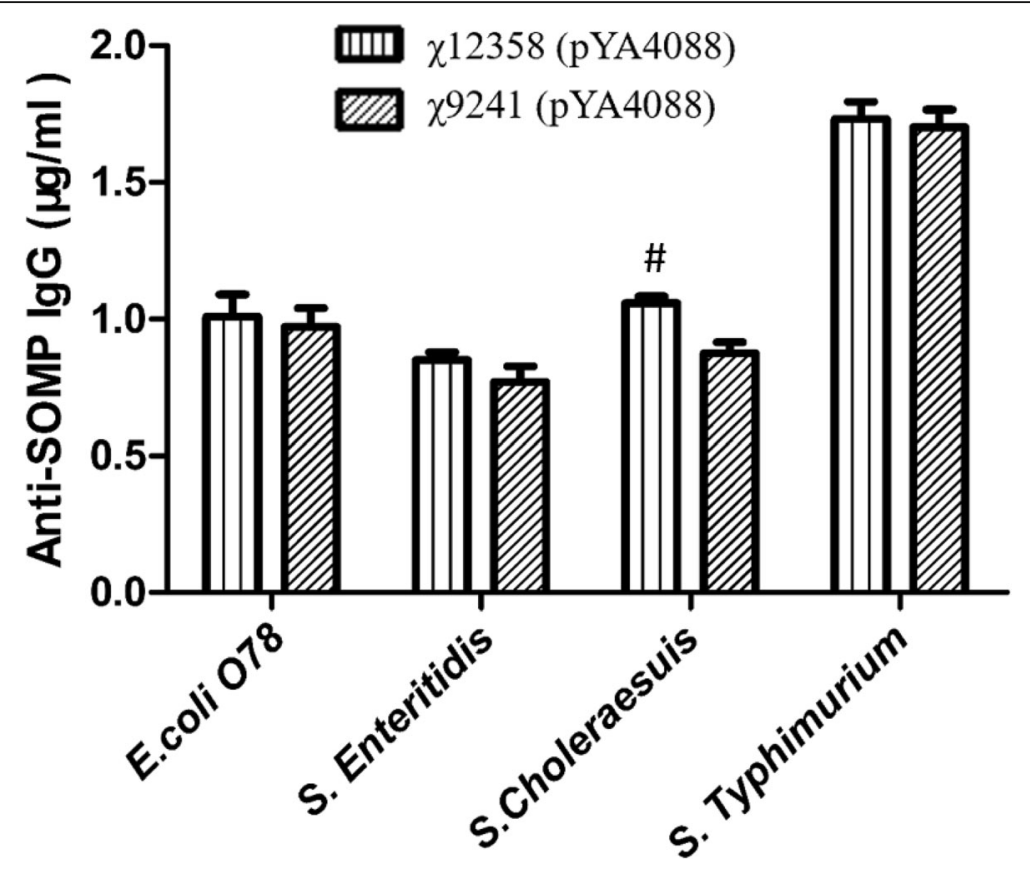

Fig. 5 Serum responses and cross-reactivity. IgG levels of sera obtained from mice orally immunized with X9241 (pYA4088) or X12358 (pYA4088) to homologous and heterologous SOMPs were measured. The error bars represented variations between triplicate wells. Mice were boosted at week 4. ${ }^{* *}, \mathrm{P}<0.01$ compared to the concentration from the mice immunized with X9241 (pYA3493). \#\#, P<0.01; \#\#\#, P<0.001 compared to the concentration from the mice immunized with X9241 (pYA4088)

control groups immunized with $X 9241$ (pYA3493) survived. However, both of the X9241 (pYA4088) and X12358 (pYA4088) strains provided significant protection against the challenge. Moreover, there was no difference in the level of protection afforded by the RASVs and wec operon mutants, suggesting that deletion of the wec operon had no influence on the protective efficacy (Table 2).

\section{Discussion}

Most mutations related to the loss of ECA are restricted to a single mutation, which are limited by the high possibility of reversible virulence and redundant genes presence in the genome, leading to a waste of energy and nutrients necessary for bacterial growth $[18,21]$. In this work, we constructed ECA-deficient S. Typhimurium mutants by deleting the entire ECA operon from wecA to $w e c G$ and evaluated the mutant strain for its virulence and ability to be used for heterologous antigen delivery.
Our studies proved the susceptibility of mutants carrying the ECA operon deletion to the bile salt deoxycholate, which is consistent with a previous report that mutations of wecA genes in Salmonella enhance the sensitivity to bile salts [11]. We also observed significant attenuation of the ECA operon mutants and persistent loading in mouse organs, consistent with previous reports $[18,21]$.

When assessing the $\mathrm{LD}_{50}$ and bacterial loading of the ECA-deficient strain in mice, we observed meningitislike symptoms (head tilt, spinning and unidirectional motion) in a small portion of the mice that were orally administered a dose higher than $10^{8} \mathrm{CFU}$ of the ECAdeficient strain $\chi^{12357}$. While the ECA-deficient strain can still potentially be used to develop vaccines [21], to ensure the safety of live Salmonella vaccines, additional genetically unlinked mutations should be included in the vaccine strain to further attenuate these potential side effects. Therefore, $\Delta$ (wecA-wecG)-6 was combined with

Table 2 Oral immunization with the PspA-expressing S. Typhimurium X9241 (pYA4088) vaccine and X12358 (pYA4088) protected $\mathrm{BALB} / \mathrm{C}$ mice against a challenge with the virulent S. pneumoniae strain WU2

\begin{tabular}{lllll}
\hline Vaccine & PspA expression $^{\text {a }}$ & Total mice/group & Survival/total $^{\text {b }}$ & Percent protection $^{\text {b }}$ \\
\hline X12358 (pYA4088) & + & 12 & $4 / 12$ & $33 \%$ \\
X9241 (pYA4088) & + & 12 & $4 / 12$ & $33 \%$ \\
X9241 (pYA3493) & - & 12 & $0 / 12$ & $0 \%$ \\
\hline
\end{tabular}

a + , PspA expressed; -, PspA not expressed

${ }^{b}$ Mice were challenged with $4 \times 10^{4}$ CFU of $S$. pneumoniae WU2 (50 times LD $_{50}$ ) 4 weeks after the second immunization 
the $\triangle p a b A B$ mutation in strain $\times 9241$ to create vaccine strain $X 12358$ to evaluate the effects of ECA deficiency on immunogenicity by introducing plasmid pYA4088, which directs the synthesis of the heterologous pneumococcal protective antigen PspA [23, 37]. The obtained vaccine strain $\chi^{12358}$ (pYA4088) constituted a regulated delayed antigen synthesis system. In the presence of arabinose, the LacI protein prevented PspA expression by binding to the $\mathrm{P}_{\text {trc }}$ promoter on pYA4088; while this strain invades and colonizes host tissues by oral immunization, LacI ceases to be synthesized due to the absence of arabinose and PspA is expressed [38].

Mice immunized with the ECA-deficient vaccine X12358 (pYA4088) elicited an anti-PspA IgG response, but the levels were significantly lower than that in mice immunized with $X 9241$ (pYA4088) (Fig. 4). The low elicited immune response by $\mathrm{X} 12358$ (pYA4088) may ascribe to its greater attenuation and low colonization in mouse tissues compared with X9241 (pYA4088) because these phenomena were also observed in the vaccine strain lacking the full-length $\mathrm{O}$-antigen [23, 37]. Therefore, it is critical for live vaccine development to achieve a proper balance between attenuation and immunogenicity through the use of new vaccine development technologies such as regulated delayed in vivo attenuation and regulated delayed in vivo antigen synthesis, which enables vaccines to efficiently colonize host tissues, thereby inducing a sufficient immune response against protective antigens synthesized in the vaccine vectors $[29,39]$. The key strategy for achieving regulated delayed in vivo attenuation relies on regulating essential gene expression by replacing the promoter of a gene of interest with the regulated promoter, such as arabinoseregulated araC $\mathrm{P}_{\mathrm{BAD}}[30,39]$. Our previous results already demonstrated that this strategy is beneficial for inducing higher immune responses against vector delivered antigens [23, 37]. Meanwhile, a Salmonella mutant $\left(\Delta r f b B 6 \quad \Delta r f G 7 \quad \Delta p a g L 73:: T T\right.$ ara $\left.C \mathrm{P}_{\mathrm{BAD}} r f b B-3\right)$, achieving the simultaneous tight regulation of both LPS and ECA synthesis in vitro by exogenously supplied arabinose, was obtained in our lab [26], and its capability to deliver heterologous antigens and induce immunogenicity in the $x 9241$ background vaccine strain will be evaluated in the future.

In mice, the direction of IgG (IgG1 vs IgG2a) switching can reflect the polarization of the Th cell response [40]. We compared the levels of two isotype subclasses of anti-PspA IgG1 and IgG2a in this study. Surprisingly, we found that the levels of the two subclasses of IgG1 and IgG2a isotype antibodies stimulated by the ECAdeficient mutant strain and its attenuated Salmonella patent strain showed different trends. The level of antiPspA IgG1 isotype antibodies in mice immunized with the ECA-deficient vaccine were significantly higher than those with $X 9241(P<0.001)$ (Fig. 4$)$, and the IgG2a levels were significantly lower than those with $X 9241$ (pYA4088) $(\mathrm{P}<0.001)$ (Fig. 4). While it is believed that murine IgG1 is strongly dependent on the IL-4 and Th2 type response, and it has a modest capacity to bind $\mathrm{C} 1 \mathrm{q}$ of the complement system due to its weak binds to Fc $\gamma$ RIIB and Fc $\gamma$ RIII, and that Th17-mediated immunity is essential for protection against pneumococcal colonization and for the clearance of colonization [41, 42], the enhanced production of IgG1 should be beneficial for preventing against pneumococcal infection and clearing extracellular pathogens because the equivalent level of protection was obtained with both the ECAdeficient strain X12358 (pYA4088) and X9241 (pYA4088), in spite of the significantly lower IgG levels induced by the ECA mutant. In addition, in this study, we employed live Salmonella as the carrier to induce an immune responses against pneumococcal surface protein antigens in a pneumococcal animal model; thus, the conclusions obtained from purified pneumococcal protective proteins or polysaccharides may not be suitable for our study as live recombinant Salmonella vaccines could stimulate all arms of the immune response, including mucosal, humoral, and cellular immunities [43, 44].

\section{Conclusions}

In summary, we examined the relationship between $S$. Typhimurium ECA and biological characteristics, virulence, colonization, immunogenicity using an ECAnegative mutant in conjunction with other attenuated mutants as vehicles for heterologous antigen delivery. Our work demonstrates that ECA affects virulence and benefits the Th2 immunity of Salmonella Typhimurium. Additionally, the ECA-deficient mutant strain of $S$. Typhimurium was seriously attenuated after oral infection [18]. In addition, our data presented here also show that the ECA mutant, in the context of an attenuated vaccine strain, induces a weak, slightly switching IgG1 immune response but still confers the same protection to $S$. pneumoniae WU2. Therefore, based on the above results, we speculate it is feasible to use a reversibly ECA mutant mode to design future Salmonella vaccine strains for heterologous protective antigens.

\section{Methods}

\section{Bacterial strains, plasmids, media and experimental animals}

The bacterial strains and plasmids used in this study are listed in Table 3. Bacterial strains were maintained in LB broth plus $20 \%$ glycerol at $-80^{\circ} \mathrm{C}$. S. Typhimurium cultures were grown at $37^{\circ} \mathrm{C}$ in $\mathrm{LB}$ broth or on LB agar. Diaminopimelic acid (DAP) $(50 \mu \mathrm{g} / \mathrm{ml})$ was added to grow the $\Delta$ asd strains [45]. LB agar containing 5\% 
Table 3 Bacterial strains and plasmids

\begin{tabular}{|c|c|c|}
\hline $\begin{array}{l}\text { Strain or } \\
\text { plasmid }\end{array}$ & $\begin{array}{l}\text { Description of the genotype or relevant } \\
\text { characteristics }\end{array}$ & $\begin{array}{l}\text { Source or } \\
\text { reference }\end{array}$ \\
\hline \multicolumn{3}{|l|}{$\begin{array}{l}\text { S. } \\
\text { Typhimurium } \\
\text { stains }\end{array}$} \\
\hline$x 3761$ & Wild type UK-1 & {$[31]$} \\
\hline x9241 & 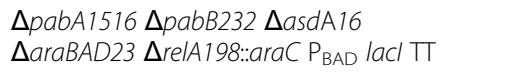 & [32] \\
\hline$\times 12357$ & $\begin{array}{l}\Delta(\text { wecA-wecG)-6, Delete whole ECA } \\
\text { operon in } \times 3761\end{array}$ & This study \\
\hline$\times 12358$ & $\begin{array}{l}\Delta \text { (wecA-wecG)-6, Delete whole ECA } \\
\text { operon in X9241 }\end{array}$ & This study \\
\hline $\begin{array}{l}\text { S. } \\
\text { pneumoniae } \\
\text { strains }\end{array}$ & $\begin{array}{l}\text { WU2, Wild-type virulent, encapsulated type } \\
3\end{array}$ & [34] \\
\hline \multicolumn{3}{|l|}{ Plasmids } \\
\hline pYA4278 & $\begin{array}{l}\text { SacB mobRP4 R6K ori } \mathrm{Cm}^{+} \text {, pRE112-T- } \\
\text { vector }\end{array}$ & {$[24]$} \\
\hline pYA5493 & Delete whole ECA operon & This study \\
\hline pYA3493 & $\begin{array}{l}\text { Plasmid } \mathrm{Asd}^{+} \text {; pBRori } \beta \text {-lactamase signal } \\
\text { sequence-based periplasmic secretion } \\
\text { plasmid }\end{array}$ & [35] \\
\hline pYA4088 & $\begin{array}{l}\text { 852-bp DNA encoding the a-helical region } \\
\text { of PspA from aa 3-285 in pYA3493 }\end{array}$ & {$[27]$} \\
\hline
\end{tabular}

sucrose was used for $s a c B$ gene-based counter-selection in the allelic exchange experiments [46]. Chloramphenicol $(\mathrm{Cm}, 25 \mu \mathrm{g} / \mathrm{ml})$ was added to the media when needed. The seven-week-old female BALB/c mice used in this experiment were purchased from Dashuo Experimental Animal Ltd. (Chengdu, China). Mice were acclimated for 7 days after arrival before starting the experiments. All animal procedures were approved by the Southwest University Animal Care and Use Committee.

\section{Plasmids and mutant strain construction}

The primers used in this study are listed in Table 4. DNA manipulations were carried out as described [47]. Transformation of $E$. coli and $S$. Typhimurium was achieved by electroporation. Transformants were selected on LB agar plates containing the appropriate antibiotics. For the ECA operon deletion experiments, Doperon-1F/Doperon-1R and Doperon-2F/Doperon-2R primers were used to amplify approximately 400-bp fragments upstream and downstream of the ECA operon from the $x 3761$ genome, respectively. The two fragments

Table 4 Primers used in this work

\begin{tabular}{ll}
\hline Primer & Sequence from 5'to 3' \\
\hline Doperon-1F & CCATCCGATGGGTGAAATTG \\
Doperon-1R & AGGGAGACCTGCAGGACGCCAGCTCCTAGAAGAGAC \\
Doperon-2F & TGGCGTCCTGCAGGTCTCCCTTCTGATGACTTGAG \\
Doperon-2R & GATATACGCCAGCAGCACTG \\
\hline
\end{tabular}

were then joined by PCR using Doperon-1F and Doperon-2R primers. Terminal A was simultaneously added to both ends of the resulting PCR product by LATaq enzyme (Takara, Japan) and ligated to pYA4278 to generate plasmid pYA5493. The mutation was introduced into $S$. Typhimurium $\times 3761$ by allelic exchange using suicide vectors pYA5493 to generate $\chi 12357$. The mutation was also introduced into $S$. Typhimurium vaccine strain $X^{9241}$ to yield strain $\chi^{12358}$.

\section{ECA western blot and LPS analyses}

ECA was prepared as previously described [48]. ECA phenotypes were characterized by immunoblotting. Bacteria grown overnight on LB plates were pelleted and washed twice with physiological saline, re-suspended in $100 \mu \mathrm{l}$ of lysis buffer $(0.065 \mathrm{M}$ pH 6.8 Tris- $\mathrm{HCl}, 5 \% \beta$ mercaptoethanol, $2 \%$ SDS, $10 \%$ glycerol, $0.05 \%$ bromophenol blue) and heated at $100{ }^{\circ} \mathrm{C}$ for $10 \mathrm{~min}$, and then incubated overnight at $60^{\circ} \mathrm{C}$ with proteinase $\mathrm{K}$ to a final concentration of $1.6 \mu \mathrm{g} / \mathrm{ml}$. Samples were boiled again for $10 \mathrm{~min}$. ECA samples were separated on $12 \%$ Tricine-SDS-PAGE gels, and transferred to nitrocellulose membranes. Membranes were probed with a 1:1000 dilution of murine anti-ECA monoclonal antibody mAb898 [48]. Blots were developed using a BCIP/NBT chromogenic substrate kit and imaged using a Gel Doc $\mathrm{TM} \mathrm{XR}+$ with Image $\mathrm{Lab}^{\mathrm{Tm}}$ Software (BIO-RAD, USA). The LPS and silver staining analyses were based on previously described protocols [49]. Briefly, cells from overnight cultures were lysed and boiled for $10 \mathrm{~min}$, and then incubated with proteinase $\mathrm{K}$ for $1 \mathrm{~h}$ at $37^{\circ} \mathrm{C}$. Subsequently, $10 \mu \mathrm{l}$ of each sample was separated by SDSPAGE (12\%), and the gels were stained with silver nitrate. For the immunoblotting experiments, the isolated ECA samples were separated by sodium dodecyl sulfatepolyacrylamide gel electrophoresis (SDS-PAGE), the resulting bands were transferred to nitrocellulose membranes, and the nitrocellulose membranes were probed with the murine anti-ECA monoclonal antibody mAb898 at a 1:1000 dilution [48].

\section{Motility assays}

LB plates containing $0.3 \%$ agar were inoculated with each strain and dried at room temperature for $2 \mathrm{~h}$. For the motility assays, three microliters of fresh bacterial liquid cultures were spotted onto the middle of plates and incubated at $37^{\circ} \mathrm{C}$ for $6 \mathrm{~h}$. The diameter of the colonies was measured. The experiments were repeated three times.

\section{DOC sensitivity assay}

Bacteria were grown until they reached an $\mathrm{OD}_{600}$ of 0.8-0.9. Dilutions from exponential cultures of each strain were spread on LB plates supplemented with $1 \%$ 
DOC [11]. Three-microliter portions of the appropriate dilutions of the exponential cultures of the wild-type and mutant strains were incubated overnight at $37^{\circ} \mathrm{C}$ in LB plates with or without $1 \%$ DOC. To precisely calculate the survival rate in DOC, $100 \mu \mathrm{l}$ of a 100 -fold dilution of the bacterial cultures with an $\mathrm{OD}_{600}$ of 0.8 were transferred to a new tube, DOC was added to final concentration of $10 \mathrm{mg} / \mathrm{ml}$, and then incubated for $1 \mathrm{~h}$ at $37^{\circ} \mathrm{C}$. The samples were then diluted to the appropriate concentration, and then $100 \mu \mathrm{l}$ of the dilutions were spread onto LB plates, followed by overnight incubation at $37^{\circ} \mathrm{C}$. The assays were repeated three times.

\section{Determination of the $\mathrm{LD}_{50}$ and colonization in mice}

Following our standard procedures, the oral $50 \%$ lethal dose (LD50) was determined [37]. Bacteria were grown statically overnight at $37^{\circ} \mathrm{C}$ in $\mathrm{LB}$ broth, diluted to $1: 50$ in fresh media, and grown with aeration at $37^{\circ} \mathrm{C}$. When the cultures reached an OD600 of 0.8-0.9, they were harvested at room temperature by centrifugation at $4000 \mathrm{rpm}$, washed once, and normalized to the required inoculum density in buffered saline gelatin (BSG) by adjusting the suspension to the appropriate OD600 value. Groups of five mice each were infected orally with $20 \mu \mathrm{l}$ containing various doses of S. Typhimurium $\times 3761$ or the mutant $\times 12357$, ranging from $1.0 \times 103 \mathrm{CFU}$ to $1.0 \times 109 \mathrm{CFU}$. Mice were observed for 4 weeks postinfection, and deaths were recorded daily. To evaluate colonization, mice were inoculated orally with $20 \mu \mathrm{l}$ of BSG containing $1 \times 109 \mathrm{CFU}$ of the wild type and mutant strains. Peyer's patches (PP), spleen and liver tissues were harvested at days $3,6,9,12,15$ and 20 postinfection. The standard procedure for determining the bacteria colonization in PP, spleen and liver in the mouse was described in our previous publications [23, 37]. Briefly, we collected all the PP from the small intestinal surface, spleen from each mouse, or cut a slice of mouse liver into $1.5 \mathrm{ml}$ tube. $1 \mathrm{ml} \mathrm{BSG}$ was added to the tube containing PP, and spleen or a slice of liver were measured the weight and extra BSG were added to a total volume of $1 \mathrm{ml}$. The samples were homogenized and plated onto LB plates to determine the number of viable bacteria after appropriate dilutions. The means of pooled PP were calculated in each mouse as bacterial colonization number in PP, and the bacterial number of liver and spleen were calculated as bacterial colonization number in spleen and liver.

\section{Western blot analyses}

To test the expression level of the heterologous antigen (PspA) delivered by the $S$. Typhimurium vaccine carrier X9241 and its ECA operon mutant strain, western blotting analyses were performed. Two proteins, LacI and GroEL, were used as the controls. The standard western blotting procedure were followed to visualize protein expression in bacteria [37], Briefly, the proteins were separated by SDS-PAGE and transferred to nitrocellulose membranes. The membranes were blocked with $3 \%$ skim milk in $10 \mathrm{mM}$ Tris and $0.9 \% \mathrm{NaCl}(\mathrm{pH} 7.4)$ and incubated with rabbit polyclonal antibodies specific for PspA, LacI or GroEL (Sigma, St. Louis, USA) [35]. Later, the membranes were washed three time using $10 \mathrm{mM}$ Tris ( $\mathrm{pH} 7.4$ ) with $0.05 \%$ Tween 20 , and inoculated in the AP-conjugated goat anti-rabbit immunoglobulin G (IgG) (Sigma) for $1 \mathrm{~h}$ at room temperature. Last, the membranes were rinsed three time with $10 \mathrm{mM}$ Tris ( $\mathrm{pH}$ 7.4) with $0.05 \%$ Tween 20 and were visualized by addition of reactive substrate of BCIP-NBT solution (Sigma), the reaction was stopped by washing the blots with large volumes of pure water when the bands developed clearly.

\section{Immunogenicity of the vaccine strains in mice}

The attenuated vaccine strains $X^{9241}$ (pYA4088), $\chi^{12358}$ (pYA4088) and $X 9241$ (pYA 3493) were cultured in LB with and without $0.1 \%$ arabinose and diluted with BSG for immunization following previous described procedures [37]. Briefly, BALB/c mice were orally inoculated with $20 \mu \mathrm{l}$ of BSG containing approximately $1.0 \times 10^{9}$ colony forming units (CFU) of each strain suspension prepared as described above, and then boosted on day 28 with the same dose of the same strain. The vaccinated mice were monitored for symptoms and mobility during the immunization period. Blood were obtained by mandibular vein puncture at biweekly intervals. Serum samples were obtained and stored at $-80^{\circ} \mathrm{C}$ for ELISA analysis.

\section{ELISA analysis of serum antibodies}

To measure the levels of antibodies triggered by the vaccine strains in the serum, ELISA assays were performed with serum antibodies against $E$. coli recombinant PspA (rPspA) and outer membrane proteins (SOMP) as previously described [37]. Briefly, rPspA and SOMP proteins of $S$. Typhimurium were prepared and purified as described previously [35]. Polystyrene 96-well flat-bottom microtiter plates were coated with $100 \mathrm{ng} /$ well of purified rPspA or SOMPs in the coating buffer $(50 \mathrm{mM}$ $\mathrm{Na}_{2} \mathrm{CO}_{3}, 50 \mathrm{mM} \mathrm{NaHCO} 3,0.1 \%$ sodium azide, $\mathrm{pH}$ 9.6), and $100 \mathrm{ng} /$ well of goat anti-mouse IgG $(\mathrm{H}+\mathrm{L})$ in PBS was applied with $100 \mu \mathrm{l}$ volumes to coat the two lines in 96-well plates for standard curve drawing. The coated plates were incubated overnight at $4{ }^{\circ} \mathrm{C}$, followed by blocking with PBS containing 10\% FBS (Sigma, USA) for $1 \mathrm{~h}$ at room temperature. A $100 \mu \mathrm{l}$ volume of serum diluted sample or mouse IgG (BD Pharmingen) for the standard curve were added to individual wells in triplicate and incubated for $1 \mathrm{~h}$ at $37^{\circ} \mathrm{C}$. Plates were treated with biotinylated goat anti-mouse IgG, IgG1 or IgG2a 
(Southern Biotechnology). Wells were developed with a streptavidin-alkaline phosphatase conjugate (Southern Biotechnology), followed by $p$-nitrophenylphosphate substrate (Sigma) in diethanolamine buffer ( $\mathrm{pH} 9.8$ ). Color development (absorbance) was recorded at 405 $\mathrm{nm}$ using a microplate reader. The standard curve was drawn using Curve Expert, and the serum antibody concentration was calculated using the standard curve.

\section{Pneumococcal challenge}

The protective efficacy of immunization with the attenuated Salmonella strains expressing PspA was assessed. Vaccinated mice at day 56 were challenged with $2 \times 10^{4}$ CFU of S. pneumoniae WU2 in $200 \mu \mathrm{l}$ of BSG by intraperitoneal (i.p.) injection and monitored and recorded daily for 15 days [34].

\section{Statistical analysis}

The antibody titer data are expressed as the geometric means, and the relative immunoreactivity is expressed as an arithmetic mean. The means were evaluated by twoway analysis of variance and chi-square test for multiple comparisons among the groups. A $P$ value of $<0.05$ was considered statistically significant.

\section{Supplementary information}

Supplementary information accompanies this paper at https://doi.org/10 1186/s12866-020-01837-0.

Additional file 1: Figure S1. The expression of PspA and lacl. The western blots showed the express of PspA in strains X12358 (pYA4088), X9241 (pYA4088) and X9241 (pYA3493). The strains were cultured in LB broth with (+) or without (-) $0.1 \%$ arabinose overnight at $37^{\circ} \mathrm{C}$. Equal numbers of cells from each strain were pelleted, suspended in protein loading buffer and boiled. Equal volumes were separated on SDS-PAGE in triplicate gels. Each gel was transferred to nitrocellulose and reacted with polyclonal antibody specific for PspA, Lacl and GroEL, respectively. GroEL was used as a standardization marker.

\section{Abbreviations}

ECA: Enterobacterial common antigen; LPS: Lipopolysaccharide; DOC: Deoxycholate; DAP: Diaminopimelic acid; BSG: Buffered saline gelatin; SDS-PAGE: Sodium dodecyl sulfate-polyacrylamide gel electrophoresis; UPEC: Uropathogenic E. coli; RASV: Recombinant attenuated Salmonella vaccine; RDES: Regulated delayed expressed system; OMP: Outer membrane proteins; BCIP-NBT: 5-bromo-4-chloro-3-indolylphosphate-nitroblue tetrazolium solution

\section{Acknowledgements}

We thank Dr. Heng Xiang of the Southwest University for bioinformatics analysis, and Ms. Chun Huang for performing the western blotting for ECA assay.

\section{Authors' contributions}

QK made conception and design of this study. QL, XS and XB conducted the experiments. QL and XS carried out the data analysis and original manuscript draft. QK reviewed and edited the manuscript writing. All authors have read and approved the manuscript.

\section{Funding}

This work is supported by the fundamental research funds for the Central Universities (SWU117061, SWU117062) and National Natural Science
Foundation of China (31970874), Chongqing natural science foundation (csct2018jcyjA1294). The funders played no role in the design of the study, analysis and interpretation of data or in writing the manuscript.

\section{Availability of data and materials \\ Not applicable.}

\section{Ethics approval and consent to participate}

This study was carried out in accordance with the recommendations of the Animal Welfare Act and US Government Principles for the Utilization and Care of Vertebrate Animals Used in Testing, Research, and Training. The protocol (2018056) for evaluating bacterial colonization, immune response and protection rate was approved by Southwest University Institutional Animal Care and Use Committee.

\section{Consent for publication}

Not applicable.

\section{Competing interests}

The authors declare that they have no competing interests.

\section{Author details}

${ }^{1}$ College of Animal Science and Technology, Southwest University, Chongqing, China. ${ }^{2}$ Chongqing Engineering Research Center for Herbivores Resource Protection and Utilization, Chongqing, China.

Received: 2 March 2020 Accepted: 1 June 2020

Published online: 08 June 2020

\section{References}

1. Bottger EC, Jurs M, Barrett T, Wachsmuth K, Metzger S, Bitter-Suermann D. Qualitative and quantitative determination of enterobacterial common antigen (ECA) with monoclonal antibodies: expression of ECA by two Actinobacillus species. J Clin Microbiol. 1987;25(2):377-82.

2. Kuhn HM, Meier-Dieter U, Mayer H. ECA, the enterobacterial common antigen. FEMS Microbiol Rev. 1988:4(3):195-222.

3. Rick PD, Mayer H, Neumeyer BA, Wolski S, Bitter-Suermann D. Biosynthesis of enterobacterial common antigen. J Bacteriol. 1985;162(2):494-503.

4. Ramia S, Amsterdam D, Mayer H, Neter E. The effect of Pseudomonas aeruginosa on the immunogenicity of enterobacterial common antigen. Immunol Commun. 1982;11(6):491-4.

5. Gozdziewicz TK, Lugowski C, Lukasiewicz J. First evidence for a covalent linkage between enterobacterial common antigen and lipopolysaccharide in Shigella sonnei phase II ECA (LPS). J Biol Chem. 2018;293(29):11652-3.

6. Bouhss A, Trunkfield AE, Bugg TDH, Mengin-Lecreulx D. The biosynthesis of peptidoglycan lipid-linked intermediates. FEMS Microbiol Rev. 2008;32(2): 208-33.

7. Lugowski C, Romanowska E. Enterobacterial common antigen: isolation from Shigella sonnei, purification and immunochemical characterization. Eur J Biochem. 1978;91(1):89-97.

8. Kiss P, Rinno J, Schmidt G, Mayer H. Structural studies on the immunogenic form of the enterobacterial common antigen. Eur J Biochem. 1978;88(1): 211-8.

9. Erbel PJA, Barr K, Gao NG, Gerwig GJ, Rick PD, Gardner KH. Identification and biosynthesis of cyclic enterobacterial common antigen in Escherichia coli. J Bacteriol. 2004;186(4):1220.

10. Mayer H, Schmidt G. Chemistry and biology of the enterobacterial common antigen (ECA). Curr Top Microbiol Immunol. 1979:85:99-153.

11. Ramos-Morales F, Prieto Al, Beuzon CR, Holden DW, Casadesus J. Role for Salmonella enterica enterobacterial common antigen in bile resistance and virulence. J Bacteriol. 2003;185(17):5328-32.

12. Inoue T, Shingaki R, Hirose S, Waki K, Mori H, Fukui K. Genome-wide screening of genes required for swarming motility in Escherichia coli K-12. J Bacteriol. 2007;189(3):950-7.

13. Castelli ME, Fedrigo GV, Clementin AL, lelmini MV, Feldman MF, Garcia Vescovi E. Enterobacterial common antigen integrity is a checkpoint for flagellar biogenesis in Serratia marcescens. J Bacteriol. 2008;190(1):213-20.

14. Neter E, Merrin C, Surgalla MJ, Wajsman Z. Shigella sonnei bacteremia. Unusual antibody response from immunosuppressive therapy following renal transplantation. Urology. 1974;4(2):198-200. 
15. Sanford BA, Thomas VL, Forland M, Carson S, Shelokov A. Immune response in urinary tract infection determined by radioimmunoassay and immunofluorescence: serum antibody levels against infecting bacterium and enterobacteriaceae common antigen. J Clin Microbiol. 1978;8(5):575-9.

16. Griffiths EK, Jewett TC, Neter E. Suppression by peritoneal irritants of antibody response to common enterobacterial antigen. J Reticuloendoth Soc. 1975;18(6):B32.

17. Chaudhuri RR, Peters SE, Pleasance SJ, Northen H, Willers C, Paterson GK, Cone DB, Allen AG, Owen PJ, Shalom G, et al. Comprehensive identification of Salmonella enterica serovar typhimurium genes required for infection of BALB/c mice. PLoS Pathog. 2009;5(7):e1000529.

18. Gilbreath JJ, Colvocoresses Dodds J, Rick PD, Soloski MJ, Merrell DS, Metcalf ES. Enterobacterial common antigen mutants of Salmonella enterica serovar Typhimurium establish a persistent infection and provide protection against subsequent lethal challenge. Infect Immun. 2012;80(1):441-50.

19. Banks KE, Fortney KR, Baker B, Billings SD, Katz BP, Munson RS, Spinola SM. The enterobacterial common antigen-like gene cluster of Haemophilus ducreyi contributes to virulence in humans. J Infect Dis. 2008;197(11):1531-6.

20. Bahrani-Mougeot FK, Buckles EL, Lockatell CV, Hebel JR, Johnson DE, Tang CM, Donnenberg MS. Type 1 fimbriae and extracellular polysaccharides are preeminent uropathogenic Escherichia coli virulence determinants in the murine urinary tract. Mol Microbiol. 2002;45(4):1079-93.

21. Bridge DR, Whitmire JM, Gilbreath JJ, Metcalf ES, Merrell DS. An enterobacterial common antigen mutant of Salmonella enterica serovar Typhimurium as a vaccine candidate. Int J Med Microbiol. 2015;305(6):511-22

22. Bridge DR, Whitmire JM, Makobongo MO, Merrell DS. Heterologous Pseudomonas aeruginosa O-antigen delivery using a Salmonella enterica serovar Typhimurium wecA mutant strain. Int J Med Microbiol. 2016;306(7): 529-40.

23. Kong Q, Liu Q, Jansen A, Curtiss R 3rd. Regulated delayed expression of rfc enhances the immunogenicity and protective efficacy of a heterologous antigen delivered by live attenuated Salmonella enterica vaccines. Vaccine. 2010;28(37):6094-103.

24. Kong Q, Yang J, Liu Q, Alamuri P, Roland K, Curtiss R 3rd. Effect of deletion of genes involved in lipopolysaccharide core and O-antigen synthesis on virulence and immunogenicity of Salmonella enterica serovar typhimurium. Infect Immun. 2011;79(10):4227-39.

25. Nagy G, Pal T. Lipopolysaccharide: a tool and target in enterobacterial vaccine development. Biol Chem. 2008;389(5):513-20.

26. Huang C, Liu Q, Luo YL, Li P, Liu Q, Kong QK. Regulated delayed synthesis of lipopolysaccharide and enterobacterial common antigen of Salmonella Typhimurium enhances immunogenicity and cross-protective efficacy against heterologous Salmonella challenge. Vaccine. 2016;34(36):4285-92.

27. Xin W, Wanda SY, Li YH, Wang SF, Mo H, Curtiss R. Analysis of type II secretion of recombinant pneumococcal PspA and PspC in a Salmonella enterica serovar Typhimurium vaccine with regulated delayed antigen synthesis. Infect Immun. 2008;76(7):3241-54.

28. Wang S, Curtiss R 3rd. Development of Streptococcus pneumoniae vaccines using live vectors. Vaccines. 2014;2(1):49-88.

29. Galen JE, Curtiss $R$ 3rd. The delicate balance in genetically engineering live vaccines. Vaccine. 2014;32(35):4376-85.

30. Clark-Curtiss JE, Curtiss R 3rd. Salmonella vaccines: conduits for protective antigens. J Immunol. 2018;200(1):39-48.

31. Hassan JO, Curtiss R 3rd. Control of colonization by virulent Salmonella typhimurium by oral immunization of chickens with avirulent cya delta crp S. Typhimurium. Res Microbiol. 1990;141(7-8):839-50.

32. Baillie LWJ, Rodriguez AL, Moore S, Atkins HS, Feng C, Nataro JR, Pasetti MF. Towards a human oral vaccine for anthrax: the utility of a Salmonella Typhi Ty21a-based prime-boost immunization strategy. Vaccine. 2008;26(48):6083-91.

33. Meier-Dieter U, Acker G, Mayer H. Detection of enterobacterial common antigen on bacterial cell surfaces by colony-immunoblotting: effect of its linkage to lipopolysaccharide. FEMS Microbiol Lett. 1989;50(1-2):215-9.

34. Briles DE, King JD, Gray MA, McDaniel LS, Swiatlo E, Benton KA. PspA, a protection-eliciting pneumococcal protein: immunogenicity of isolated native PspA in mice. Vaccine. 1996;14(9):858-67.

35. Kang HY, Srinivasan J, Curtiss R 3rd. Immune responses to recombinant pneumococcal PspA antigen delivered by live attenuated Salmonella enterica serovar typhimurium vaccine. Infect Immun. 2002;70(4):1739-49.

36. Nagy G, Palkovics T, Otto A, Kusch H, Kocsis B, Dobrindt U, Engelmann S, Hecker M, Emody L, Pal T, et al. "Gently rough": the vaccine potential of a
Salmonella enterica regulatory lipopolysaccharide mutant. J Infect Dis. 2008; 198(11):1699-706.

37. Kong Q, Liu Q, Roland KL, Curtiss R 3rd. Regulated delayed expression of $\mathrm{rfaH}$ in an attenuated Salmonella enterica serovar typhimurium vaccine enhances immunogenicity of outer membrane proteins and a heterologous antigen. Infect Immun. 2009;77(12):5572-82.

38. Wang S, Li Y, Scarpellini G, Kong W, Shi H, Baek CH, Gunn B, Wanda SY, Roland $\mathrm{KL}$, Zhang $\mathrm{X}$, et al. Salmonella vaccine vectors displaying delayed antigen synthesis in vivo to enhance immunogenicity. Infect Immun. 2010; 78(9):3969-80.

39. Wang S, Kong Q, Curtiss R 3rd. New technologies in developing recombinant attenuated Salmonella vaccine vectors. Microb Pathogenesis. 2013;58:17-28.

40. Zhang Y, Dominguez-Medina C, Cumley NJ, Heath JN, Essex SJ, Bobat S, Schager A, Goodall M, Kracker S, Buckley CD, et al. lgG1 is required for optimal protection after immunization with the purified porin OmpD from Salmonella typhimurium. J Immunol. 2017;199(12):4103-9.

41. Bournazos S, DiLillo DJ, Ravetch JV. The role of fc-FcgammaR interactions in IgG-mediated microbial neutralization. J Exp Med. 2015;212(9):1361-9.

42. Voss F, Kohler TP, Meyer T, Abdullah MR, van Opzeeland FJ, Saleh M, Michalik S, van Selm S, Schmidt F, de Jonge MI, et al. Intranasal vaccination with lipoproteins confers protection against Pneumococcal colonisation. Front Immunol. 2018;9:2405.

43. Curtiss R, Hassan JO. Nonrecombinant and recombinant avirulent Salmonella vaccines for poultry. Vet Immunol Immunop. 1996;54(1-4):365-72.

44. Medina E, Guzman CA. Use of live bacterial vaccine vectors for antigen delivery: potential and limitations. Vaccine. 2001;19(13-14):1573-80.

45. Nakayama KK, SM; Curtiss, R: Construction of an Asd+ expression-cloning vector: stable maintenance and high level expression of cloned genes in a Salmonella vaccine strain. Nat Biotechnol 1988;6:693-7.

46. Kang HY, Dozois CM, Tinge SA, Lee TH, Curtiss R 3rd. Transduction-mediated transfer of unmarked deletion and point mutations through use of counterselectable suicide vectors. J Bacteriol. 2002;184(1):307-12.

47. Sambrook J, Fritsch E, Maniatis T. Molecular cloning: a laboratory Manua I. Cold Spring Harbor: Cold Spring Harbor Laboratory Press; 1989.

48. Peters $H$, Jurs M, Jann B, Jann K, Timmis KN, Bittersuermann D. Monoclonal antibodies to enterobacterial common antigen and to Escherichia coli lipopolysaccharide outer core - demonstration of an antigenic determinant shared by enterobacterial common antigen and Escherichia coli K5 capsular polysaccharide. Infect Immun. 1985;50(2):459-66

49. Hitchcock PJ, Brown TM. Morphological heterogeneity among Salmonella lipopolysaccharide chemotypes in silver stained polyacrylamide gels. J Bacteriol. 1983;154(1):269-77.

\section{Publisher's Note}

Springer Nature remains neutral with regard to jurisdictional claims in published maps and institutional affiliations.

Ready to submit your research? Choose BMC and benefit from

- fast, convenient online submission

- thorough peer review by experienced researchers in your field

- rapid publication on acceptance

- support for research data, including large and complex data types

- gold Open Access which fosters wider collaboration and increased citations

- maximum visibility for your research: over $100 \mathrm{M}$ website views per year

At BMC, research is always in progress.

Learn more biomedcentral.com/submissions 